\title{
INTERFACES HÁPTICAS: SISTEMAS CINESTÉSICOS VS. SISTEMAS TÁCTILES
}

\section{Vera Zasúlich PÉrez ARIZA ${ }^{1}$ MAURICIO SANTÍS-CHAVES ${ }^{2}$}

\section{INTRODUCCIÓN}

El sentido háptico complementa el sentido de la vista porque permite obtener información de otras características físicas y con ello proporciona, a través de movimientos de actividad exploratoria, mayor detalle del objeto con el que un usuario interactúa (Do et al., 2012).

El término háptico (del griego háptō/ haptesthai (tocar, relativo al tacto) es el adjetivo usado para describir lo relacionado con o basado en el sentido del tacto, además que hace referencia a la ciencia que estudia todo lo relativo al tacto y sus sensaciones como medio de control e interacción con máquinas y computadores (González, 2011; Genoy Muñoz et al., 2011). La información del sentido háptico proviene de la manipulación activa y voluntaria de objetos en el entorno hecha por los dedos y las manos, e involucra el sentido del tacto y la percepción del movimiento del cuerpo que se conoce como cinestesia (Cortés et al., 2010). Por lo general para la manipulación de un objeto, el aspecto táctil se refiere a lo estático, y es la información recibida de las terminales nerviosas de la piel, mientras que lo cinestésico se relaciona con lo dinámico de dicha interacción con el objeto (Carter y Fourney, 2005).
En consecuencia, una interfaz háptica (IH) es aquel dispositivo que se encarga de reproducir en el usuario lo captado por la sensación de contacto y manipulación de un objeto que se encuentra dentro de un ambiente virtual o en un entorno remoto, simulando las características esenciales al tacto y sujeción de un objeto real, tales como temperatura, textura, peso, contorno, entre otros (Golledge et al., 2006; Hernantes et al., 2012).

Existe un número considerable de dispositivos hápticos que permiten a los usuarios que estén distanciados intercambiar información a través del sentido del tacto. Esta información puede ser percibida de objetos inanimados o dispositivos controlables (Rantala et al., 2011).

El sentido háptico abarca dos tipos de sensaciones que producen información de un objeto y que son útiles para la interpretación que el cerebro humano hace de este. Tiene que ver con el rasgo percibido del cuerpo manipulado. Si es su masa, esta provee información del peso e inercia, se tiene idea por medio del sentido cinestésico a través de receptores ubicados en los músculos, las articulaciones y los tendones; estos receptores le permiten también a la persona sentir las fuerzas-torques ejercidas al contacto con un cuerpo y saber en dónde se encuentra su mano en el espacio, aunque sea con los ojos

1 Ingeniera Electrónica, PhD. Docente titular Facultad de Ingenierías, Línea Biomecatrónica, Grupo de Investigaciones Centro de Bioingeniería, Universidad Pontificia Bolivariana, Medellín.

1 Ingeniero Electrónico, Especialista en Control e Instrumentación Industrial. Asistente de investigación, Línea Biomecatrónica, Grupo de Investigaciones Centro de Bioingeniería, Universidad Pontificia Bolivariana, Medellín.

Autor de correspondencia: Pérez-Ariza, V. (Vera). Universidad Pontificia Bolivariana: Campus de Laureles Circular 1 No. 73-76. Teléfono: (4) 4488388 ext 12401. Correo electrónico: vera.perez@upb.edu.co
Historia del artículo:

Artículo recibido: 9-III-2016 / Aprobado: 11-XI-2016

Disponible online: 30 de febrero de 2017

Discusión abierta hasta abril de 2018 
cerrados (Coles, 2011); desde el punto de vista fisiológico, lo cinestésico tiene que ver con estar consciente de la posición y del movimiento del cuerpo en el espacio, es la parte del sistema somatosensorial que es consciente de la percepción corporal y se encuentra distribuida en todo el cuerpo (Fogtmann et al., 2008). Si el estímulo es de textura, temperatura, presión o vibración, se relacionan directamente con los mecanorreceptores cutáneos o táctiles situados en la piel (Chen et al., 2006); la piel glabra o sin pelo (manos, labios y pies) es la que más responde al tacto respecto a las partes que sí tienen pelos (Nakamura et al., 2003).

De manera análoga, las IH pueden dividirse en dos grupos principales desde el punto de vista de la sensación que producirán en el momento del contacto con la parte del cuerpo: las que producen estímulos cinestésicos y las que producen estímulos táctiles. Existen varias IH ya desarrolladas y otras en etapa de investigación (Bilgincan et al., 2010; Ferre et al., 2008). El primer tipo de interfaz apunta a estímulos de fuerza en las extremidades (dureza, peso e inercia)(Vélez, 2011), siendo complementado por algoritmos de "representación" (rendering) háptica que calculan las fuerzas de interacción entre el dispositivo y los objetos virtuales que manipula (Nájera y Díaz, 2005). El segundo tipo va enfocado a un actuador que estimula la piel en cualquier zona del cuerpo, aplicando señales de temperatura, vibración, presión, o rugosidad, entre otros (Vélez, 2011).

El grado de realismo con el que el usuario percibe el estímulo que recrea el objeto virtual se debe en gran medida a la tecnología que emplea el dispositivo con el que interactúa, al tipo de actuación sobre el cuerpo humano (cinestésico o táctil) y a la acción complementaria de un algoritmo-programa que ayude a la recreación del estímulo (Lim et al., 2014). Por tanto, la especificidad y el grado de realismo que tengan los dispositivos hápticos que se encuentran en el mercado, influirá en los costos de adquisición, en el usuario final y en el tipo de aplicación.

El desarrollo del presente artículo parte de la observación y se apoya en el estado del arte para de- mostrar una tendencia en combinar sistemas cinestésicos con sistemas táctiles para lograr mayor realismo en la percepción de las sensaciones y pretende plantear un análisis de este fenómeno. La sección 2 presenta las características de los sistemas cinestésicos incluyendo su principio de funcionamiento, evolución histórica, tecnologías comúnmente utilizadas para su construcción y casos específicos. La sección 3 presenta un desarrollo análogo para el caso de los sistemas táctiles. La sección 4 presenta el estado de difusión comercial de ambos tipos de sistemas. La sección 5 muestra ejemplos de combinar los dos tipos de interfaces existentes. La sección 6 plantea una discusión, teniendo en cuenta la información planteada en las secciones anteriores, y por último la sección 7 presenta las conclusiones.

\section{IH DE TIPO CINESTÉSICO}

Una IH cinestésica actúa sobre los aspectos activos del tacto (entendida como la información adquirida mediante los movimientos y/o la fuerza en músculos y articulaciones) (Carter y Fourney, 2005).

Esto conlleva una realimentación de fuerzas por parte de la IH cinestésica y ocurre por el intercambio de fuerzas entre el usuario y el ambiente virtual en el que se encuentre. Para lograr "inmersión corporal" (embodiment) la interface deberá ser capaz de afectar al cuerpo o la parte en contacto, para simular el peso de objetos alzados (Bergamasco y Ruffaldi, 2011). Este principio y su mejora se aprovechan al máximo en el desarrollo de juegos serios y procesos de rehabilitación con dispositivos hápticos-cinestésicos tipo robot (Bouri et al., 2013).

La tecnología que utilizan las interfaces cinestésicas busca ejercer una realimentación de fuerza controlada sobre la parte del cuerpo, normalmente los dedos, la palma de la mano o el brazo, para recrear al objeto (Bergamasco y Ruffaldi, 2011). Esto conlleva a que su principio activo sea de tipo electromecánico y puede ser logrado por varios métodos, utilizando metales con memoria de forma, motores eléctricos en continua, cilindros neumáticos, actuadores magnéticos, en conjunto con diferentes 
aparejos mecánicos, como articulaciones, bandas, guayas, que trasladen la fuerza producida y focalicen su efecto puntualmente en la extremidad del usuario (Youngblut et al., 1996).

Las IH cinestésicas o de realimentación de fuerzas muestran un comportamiento semejante al de mecanismos robots con los que el usuario interactúa e intercambia energía mecánica (O’Malley y Gupta, 2008). Estas tienen en cuenta los grados de libertad (Degrees of Freedom, DOF) con que el usuario puede moverse usando el dispositivo, lo que acarrea mayor naturalidad en la interacción realizada. Tres grados de libertad implica que el movimiento puede ser en tres dimensiones, pero al aumentar su cantidad repercute en disminución en la restricción del movimiento, dando mayor capacidad al espacio de trabajo y aumento de la naturalidad en la ejecución de la interacción. Las IH cinestésicas de 3 a 6 DOF son las más difundidas, debido a que tanto en el aspecto mecánico como de programación son relativamente sencillas de desarrollar, van de la mano con un bajo costo de producción y tienen versatilidad en su manejo (ElSaddik et al., 2011).

Las IH deben tener en cuenta las limitaciones funcionales dadas por el equilibrio entre el diseño y la versatilidad en su desempeño, esto es, a mayor espacio de trabajo requerirá mayor longitud de los elementos, mayor resistencia en el material - comúnmente metal一, que la hará más pesada, lo que aumenta la inercia y a su vez disminuye la rigidez de la interfaz (López et al., 2011). De igual forma, a mayor número de articulaciones, también la haría voluminosa y de compleja controlabilidad. Si bien esto proporciona retos en el diseño, en la actualidad se ha optado por implementaciones de las piezas en materiales novedosos-alternos a base de carbono, como los polímeros y otros compuestos (O'Malley y Gupta, 2008), además de una combinación estratégica con el metal, lo cual permitirá diseños rígidos y livianos.

Otro aspecto que caracteriza a una IH cinestésica es su portabilidad: las hay de escritorio (joystick), y según el tipo de anclaje o soporte pueden ser portátiles o fijas. Las de tipo portable están monta- das y sujetas al cuerpo del usuario por lo que este puede mover la extremidad y desplazarse, esto conlleva a que se tenga una referencia relativa para las fuerzas ejercidas. El otro tipo de anclaje es en el que la interfaz se halla sujeta a una base empotrada en un lugar fijo, ya sea escritorio, piso, pared o techo, por lo que el usuario debe ubicarse en un lugar específico y por lo general mover sólo la extremidad sujeta a la interfaz (Sabater, 2003).

El tipo de efector final puesto a la interfaz fija ayuda al realismo que esta propicia en la interacción con el ambiente virtual o físico que posea. Para el caso de la mano humana es muy diferente un efector de una interfaz tipo tijera o de pinza, que se sujeta con dos o más dedos específicos así como de los músculos que involucra, al que tiene otra interfaz con la forma de un lapicero o un instrumento similar como un bisturí (Wagner, 2014).

La forma en que mecánicamente se construyen las IH cinestésicas se define en dos grandes categorías relacionadas con la forma en que se le aplica la realimentación de fuerza: por elementos enlazados y por elementos tensionados. Los primeros son de tamaño reducido y portables, mientras que los otros son de gran tamaño. La interfaz más comúnmente utilizada es la de elementos enlazados, en donde elementos rígidos se enlazan entre sí hasta el efector final y son activados por motores eléctricos situados en la base del dispositivo; esta configuración provee una buena transmisión y trazado de la fuerza hacia el efector final. La fuerza del motor será mayor a medida que aumente el peso y el largo del enlace, además que se requerirá de codificadores de posición de mayor precisión si se necesita movilidad en un espacio de trabajo reducido (Coles, 2011). Ejemplo de estas son Novint Falcon (Novint, 2012), Geomagic (Geomagic, 2015) y Phantom (Sensable, 2016).

El segundo grupo de interfaces tiene como principio la transferencia de la fuerza a través de cables tensionados, esto es, que mediante poleas y guías el efector final recibe la fuerza ejercida por motores de continua y se gradúa su movimiento mediante codificadores digitales conectados a ellos. 
El ejemplo de este tipo de interfaz es el SPIDAR (Sato, 2002), básico y modelos posteriores. En esta los elementos se montan en una estructura cúbica o cilíndrica y en ella se distribuyen los motores, unos arriba y otros en la parte baja tensionando en oposición, con los apoyos de los cables en las esquinas. En la parte central del espacio de trabajo está suspendido el efector en equilibrio para apoyar el dedo o la mano y es tensionado según la interacción que realice. Con este diseño se pueden tener desde uno a seis grados de libertad, y se infiere que a mayor número de cables se tendría mayor fidelidad en la fuerza ejercida (Coles, 2011). Este dispositivo se ha escalado a dimensiones promedio de una persona adulta para diversas aplicaciones en la enseñanza y el diseño (Naud et al., 2009).

En este segundo grupo se encuentra también el exoesqueleto háptico, el cual es otra clase de interfaz para transferencia de fuerza que ha sido desarrollada tiempo atrás, y se basa en cables tensionados por motores y codificadores de posición. El soporte de estos dispositivos puede estar en el suelo, la pared, un escritorio, o en el cuerpo del usuario y pueden lograr más de seis grados de libertad en el área de trabajo (Sabater, 2003). Comúnmente existen sistemas de sujeción en dedos y manos (Torres, 2012) o brazos (Sledd y O'Malley, 2006) y su aplicación también se extiende al campo de la rehabilitación y la ergonomía. Por ejemplo, el exoesqueleto que va sujeto al brazo busca reproducir agarre y sujeción por medio del apoyo de las fuerzas en la mano y a dos o varios dedos, ejemplo de exoesqueletos comerciales son la interfaz Cybergrasp (Cyberglovesystems, 2015a) y la interfaz HIRO III (Robothand, 2015).

Existen IH cinestésicas que utilizan un principio de realimentación de fuerza diferente a las técnicas y métodos clásicos. Un ejemplo de ello es el tipo de interfaz cinestésica cuyo principio de funcionamiento es por medio de campos magnéticos fuertes, empleando el principio de levitación de Lorentz para la interacción de fuerzas (Berkelman y Dzadovsky, 2010), tal como se materializa en el sistema comercial Maglev 200 ${ }^{\mathrm{TM}}$ (Butterfly-haptics, 2015).
La Tabla 1 resume las tecnologías comunes utilizadas para el desarrollo de las IH cinestésicas (Ueberle, 2006).

\begin{tabular}{|c|c|c|}
\hline Tecnología & $\begin{array}{l}\text { Mecanismo } \\
\text { actuador }\end{array}$ & Zona de contacto \\
\hline Neumático & Pistón & $\begin{array}{l}\text { Apoyado directo a } \\
\text { las extremidades }\end{array}$ \\
\hline Hidráulico & Pistón & $\begin{array}{l}\text { Apoyado directo a } \\
\text { las extremidades }\end{array}$ \\
\hline Eléctrico & $\begin{array}{l}\text { Motor DC conecta- } \\
\text { do directamente o } \\
\text { por cables y poleas }\end{array}$ & $\begin{array}{l}\text { Brazo, muñeca- } \\
\text { mano o dedos }\end{array}$ \\
\hline Magnético & $\begin{array}{l}\text { Elemento en levi- } \\
\text { tación por el prin- } \\
\text { cipio de Lorentz }\end{array}$ & Mano, dedos \\
\hline
\end{tabular}

\section{IH DE TIPO TÁCTIL}

La IH tipo táctil, también conocida como pantalla táctil, es un dispositivo que se encarga de estimular los receptores nerviosos del tacto para desplegar en la interacción con la piel humana parámetros como temperatura, rugosidad, forma y textura. Los mecanorreceptores que comúnmente se estimulan en las pantallas táctiles y logran simular contacto en la piel son los de vibración y presión, dado que con la presión se activan los discos de Merkel y con una vibración de baja o alta frecuencia se activan los corpúsculos de Meissner y de Pacini, respectivamente (Chouvardas et al., 2008). En varios estudios se ha podido demostrar que la realimentación táctil en la yema de los dedos tiene el potencial de aumentar el grado de inmersión en el usuario en ambientes virtuales o remotos (telepresencia y teleoperación) (García-Hernández et al., 2014).

También se han desarrollado interfaces electrocutáneas, usando el principio de electroestimulación de las terminales nerviosas a nivel de superficie de la piel; estas interfaces tienden a ser pequeñas, durables, eficientes y son libres de resonancia mecánica (Kajimoto et al., 2004; Sato y Tachi, 2010). Este método de estimulación electro-táctil puede 
producir una amplia variedad de sensaciones en la piel, desde un leve hormigueo hasta golpes dolorosos, siempre que se varíe la frecuencia y la amplitud de los pulsos aplicados sobre la misma (Pamungkas y Ward, 2015). También se han implementado interfaces de tipo electrostático, que recrean la sensación de fricción por medio de la generación de fuerzas normales entre la piel del dedo y la pantalla, tal como ocurre entre las placas de un condensador (Xu et al., 2011).

Para simular contacto por presión, por lo general, se utilizan dispositivos de gran tamaño que contrastan con la pequeña porción de piel sobre la cual actúan, por ejemplo en la yema de los dedos. La tecnología del principio activo de estimulación se materializa en grandes dimensiones, por la cantidad de elemento activos que conlleva un consumo de corriente elevado y en ocasiones dificulta su portabilidad. Ejemplo de esta apreciación se puede evidenciar en interfaces para un dedo que generan estímulo de tipo Braille, que para impulsar las agujas o pines usan servomotores (Wagner et al., 2004), y otros que plantean una matriz táctil, impulsada en un primer desarrollo por una bola fija que se traslada en dos dimensiones y en un segundo desarrollo por efecto neumático con tres bits de resolución (Benali-Khoudja et al., 2004).

Para simular textura se utilizan dispositivos electromecánicos de reducido tamaño como los vibradores-resonadores de inercia, lineales (Linear Resonator Actuator, LRA) y los motores con masa excéntrica rotativa (Eccentric Rotating Mass, ERM) (Yang, 2013; Wang, 2014), al igual que los LRA de impacto (Pyo et al., 2015); otros de tamaño mediano como las bobinas de voz (Richter et al., 2011), y algunas de gran tamaño que ofrecen mayor resolución como pantallas táctiles gráficas a base de matriz de agujas activadas por solenoides (Simeonov y Simeonova, 2014), por servomotores (Wagner et al., 2004), o que usan barras largas de piezoeléctricos dimorfos en una interfaz tipo Braille (Lee y Lucyszyn, 2005). Hay un desarrollo particular que recrea una pantalla táctil dinámica-refrescable tipo
Braille, por medio de novedosas válvulas neumáticas que se comportan como un biestable (activo e inactivo), las cuales se mantienen en una posición y producen una protuberancia por la salida de aire continua bajo una membrana, y al desactivarse cierra el paso del aire, por lo que desaparece dicha protuberancia (Russomanno et al., 2015).

En general, el estímulo táctil se puede obtener de diversas formas y las tecnologías que han sido usadas comúnmente para generarlos son la neumática, solenoides, resonadores piezoeléctricos, bobina de voz, alambres de memoria de forma, banda tensionada por motor y la bomba de calor, entre otras. Dichas tecnologías son resumidas en la Tabla 2 (Pasquero, 2006), teniendo en cuenta que a la fecha no ha habido cambios significativos.

Adicionalmente, en el desarrollo de algunas interfaces táctiles, es importante considerar otras características de diseño. Algunos tipos de actuadores involucran el concepto de bajo consumo de energía, como por ejemplo los de lámina de cerámica piezoeléctrica, mono o multicapa (Poupyrev et al., 2002). Otros desarrollos contemplan la adecuada ubicación de los actuadores sobre la superficie de la piel que se vaya a estimular, la resistencia a la suciedad, las secreciones de la piel y el roce con esta. De igual manera es una importante consideración de diseño el hecho de que hay que hacer refrescoactualización de la impresión táctil hecha a una tasa de $1 \mathrm{KHz}$ (Pasquero y Hayward, 2003) o un valor menor cercano $(690 \mathrm{~Hz})$ que sea práctico y efectivo según la aplicación realizada (Lévesque et al., 2012).

Las IH táctiles han tenido desarrollos tendientes a encontrar un equilibrio entre el costo, la portabilidad de los dispositivos y la sensación producida. En este sentido se han realizado diseños prometedores que apuntan a lo portable, de tamaño reducido, que se sujeta directamente en el dedo, el cual imprime un estímulo de contacto a la yema del dedo por medio de una plataforma móvil, que se mueve respecto al soporte y ofrece la sensación de contacto con una superficie orientada arbitrariamente, con 2 DOF (Yazdian et al., 2013) y con 3 DOF (Chinello et al., 2015). 
Teniendo en cuenta todo lo anterior, el avance de las interfaces táctiles ha sido lento y los sistemas desarrollados desde principio de siglo han sido voluminosos, a lo que se suma que algunos carecen de la portabilidad necesaria para adaptarse a una $\mathrm{IH}$ de fuerza para crear una completa realimentación háptica (Coles et al., 2011), otros son costosos y frecuentemente optimizados hacia una sola característica de sensación reproducida, por lo que si se desea un desempeño más realista, el dispositivo deberá cumplir con un conjunto de exigencias que le permitan proporcionar una variedad de sensaciones al usuario (Pasquero y Hayward, 2003). El análisis de las características generales de las IH táctiles actuales muestra que las que dan muy buena resolución son voluminosas y muestran limitaciones de usabilidad (Fontana et al., 2012).

Esta apreciación se puede evidenciar en dos dispositivos recientes que generan estímulos de textura y suavidad al apoyarse en la yema del dedo. El primero, de escritorio, simula suavidad al contacto; se basa en una banda de tejido elástico que es soportada por dos servomotores, cuyo movimiento coordinado determina una traslación si giran en un mismo sentido o una tensión-relajación si giran en sentido opuesto (Bianchi y Serio, 2015). El segundo dispositivo tiene actuador de vibración sobre un disco en contacto con el dedo que está soportado en una estructura móvil que genera dos grados de libertad angulares y uno traslacional. Esto proporciona un movimiento que le permite tener la sensación de una superficie plana, curva y de un borde (Pérez et al., 2015), este dispositivo si bien es portable se dificulta tenerlo en dos dedos consecutivos.

Otro dispositivo que utiliza el mismo principio de dos motores y una banda elástica, es el diseñado y construido para simular caricias en el antebrazo de un usuario, el cual dentro de sus resultados contempló el agrado y desagrado de la sensación producida, y plantea considerar más a fondo también la diferencia de sexo para la extracción de características de la señal obtenida en el sujeto de prueba (Bianchi et al., 2014).

\section{TABLA 2. RESUMEN DE LAS TECNOLOGÍAS COMUNES PARA IH TÁCTILES}

\begin{tabular}{|c|c|c|}
\hline Tecnología & Descripción & Sensación \\
\hline $\begin{array}{l}\text { Electrostático } \\
\text { (Xu et al., 2011) }\end{array}$ & $\begin{array}{l}\text { Capacitor con aislante de Polimida, formado por los fluidos } \\
\text { conductores del dedo que actúan como una placa y un electrodo } \\
\text { externo que actúa como la otra placa. }\end{array}$ & $\begin{array}{l}\text { Fricción, reproduce fuerzas de } \\
\text { corte en la piel }\end{array}$ \\
\hline $\begin{array}{l}\text { Electrocutáneos (Kajimoto et } \\
\text { al., 2004; Sato y Tachi, 2010) }\end{array}$ & $\begin{array}{l}\text { Se hace electroestimulación sobre la piel para activar las } \\
\text { terminaciones nerviosas. }\end{array}$ & Presión y vibración suave \\
\hline Vibro-táctil & $\begin{array}{l}\text { Motor DC con masa excéntrica sujeta al eje de giro (Yang, 2013). } \\
\text { Actuador Lineal de Resonancia (Linear Resonator Actuator, LRA) } \\
\text { (Wang, 2014). } \\
\text { Actuador Lineal de Resonancia de Impacto (Linear Impact } \\
\text { Resonator Actuator, LIRA) (Pyo et al., 2015) }\end{array}$ & Vibración por pulso o sostenida \\
\hline $\begin{array}{l}\text { Vibro-resonancia } \\
\text { (Kyung y Kwon, 2008) }\end{array}$ & Vibro-resonador piezoeléctrico de barras paralelas & $\begin{array}{l}\text { Vibración con gama de } \\
\text { frecuencias de resonancia }\end{array}$ \\
\hline $\begin{array}{l}\text { Sistema de despliegue de } \\
\text { temperatura desplazada (DTSS) } \\
\text { (Ferre et al., 2008) }\end{array}$ & Calefactor puesto en un dedal & Cambios de temperatura \\
\hline Presión Neumática & Pistón neumático sujeto a la mano & $\begin{array}{l}\text { Contacto en el dedo de una } \\
\text { superficie o patrón en ella }\end{array}$ \\
\hline Solenoide & Pistón electromagnético & Presión al sujetar \\
\hline
\end{tabular}




\begin{tabular}{|c|c|c|}
\hline $\begin{array}{l}\text { Exoesqueleto para múltiples } \\
\text { dedos }\end{array}$ & Armazón que conecta un actuador de presión en cada dedo & $\begin{array}{l}\text { Fuerza de agarre, presión, peso } \\
\text { sostenido }\end{array}$ \\
\hline Presión directa en el dedo & $\begin{array}{l}\text { Correa o placa que se ajusta o relaja en la punta del dedo me- } \\
\text { diante uno o varios motores DC }\end{array}$ & $\begin{array}{l}\text { Presión de contacto sostenida, } \\
\text { gradual, vibración, curvatura, } \\
\text { borde }\end{array}$ \\
\hline $\begin{array}{l}\text { Polímero Electro Activo (Electro } \\
\text { Active Polymer, EAP) (Bolz- } \\
\text { macher et al., 2004; Runyan y } \\
\text { Blazie, 2010; Pei et al., 2009) }\end{array}$ & $\begin{array}{l}\text { Material polimérico químicamente modificado para que un estí- } \\
\text { mulo físico lo active, dado por un alto voltaje aplicado }\end{array}$ & $\begin{array}{l}\text { Presión por abultamiento super- } \\
\text { ficial, textura rugosa, patrones en } \\
\text { superficie }\end{array}$ \\
\hline Agujas de presión & $\begin{array}{l}\text { Conjunto de agujas movidas por campo electromagnético que } \\
\text { simulan presión y patrones para sistema Braille } \\
\text { Agujas movidas por Alambre con memoria de Forma (Shape Me- } \\
\text { mory Alloy, SMA) (Kontarinis et al., 1995). }\end{array}$ & Presión sostenida, pulsos suaves \\
\hline Térmicos & $\begin{array}{l}\text { Se basa en una placa de Peltier que controla la temperatura que } \\
\text { la interfaz transfiere a la piel. }\end{array}$ & $\begin{array}{l}\text { Calentamiento en una zona redu- } \\
\text { cida de la piel }\end{array}$ \\
\hline $\begin{array}{l}\text { Cerámico piezoeléctrico } \\
\text { (Poupyrev et al., 2002) }\end{array}$ & $\begin{array}{l}\text { Lámina mono o multicapa de cerámico piezoeléctrico que oscila } \\
\text { por voltaje aplicado. }\end{array}$ & $\begin{array}{l}\text { Vibración sostenida o por pulsa- } \\
\text { ciones }\end{array}$ \\
\hline $\begin{array}{l}\text { Fluido reológico } \\
\text { (Song et al., 2003) }\end{array}$ & $\begin{array}{l}\text { Fluido que modifica su viscosidad mediante la aplicación de un } \\
\text { campo eléctrico o magnético. }\end{array}$ & Rigidez \\
\hline
\end{tabular}

Se ha desarrollado una IH táctil tipo lapicero, conocido como UbiPen, el cual en un mismo dispositivo agrupa un motor de vibración y una matriz de agujas como pantalla táctil, en donde cada aguja es activada por un motor lineal ultrasónico; esta combinación genera patrones de vibración y de textura en la mano del usuario (Evreinova et al., 2014).

Existen en desarrollo IH táctiles con un principio activo diferente de los convencionales que permiten dar la sensación de suavidad. Entre estos están las de fluido Reorológico (Song et al., 2003), las cuales se encapsulan en un pistón y al aplicarles un voltaje modifican su estructura pasando de líquido a sólido y se opone así al movimiento. Las de fluido Magnetoreológico, que actúan igual que el anterior, pero al ser sometidos a un campo magnético (Pasquero, 2006).

Otro tipo de actuador que reproduce sensación de contacto suave por presión se constituye de Polímero Electroactivo (Electro Active Polymer, EAP) (Matysek et al., 2009), el cual presenta un cambio de volumen al aplicarle un campo eléctrico de alto valor, del orden de los kilovoltios. El EAP se encuentra en forma de gel, el cual se encapsula para aislarlo (Bolzmacher et al., 2004). Estos actuadores también se han aplicado para la activación de interfaces portables tipo Braille, que reproducen forma (Pei et al., 2009; Runyan y Blazie, 2010).

Por otra parte se encuentran en desarrollo interfaces ultrasónicas, las cuales generan presión en la piel en forma distribuida por medio de frentes de onda a nivel de ultrasonido, liberando al usuario de colocarse cualquier dispositivo en las manos (Iwamoto y Shinoda, 2005; Hoshi et al., 2010). Existe un tipo de interfaz que también trabaja con el mismo principio de presión de aire sobre la piel del dedo, la cual es controlada por apertura o cierre en la salida del aire (Bianchi et al., 2011). Además están también las de chorro de agua, que lanzan agua a presión al dedo cuando pasa por un área determinada para simular el contacto con un objeto representado en dicha área (Richter et al., 2013).

Una derivación de IH táctil que usa presión de aire es la de tipo de lámina deslizante sobre aire, la cual da la sensación de fricción y se basa en que sobre una superficie vibrante se le ubica encima una lámina en donde se coloca el dedo, que queda suspendida en un colchón de aire debido a la vibración ultrasónica de dicha superficie. Con el aumento o 
disminución de la vibración, disminuirá o aumentará la fricción con la lámina (Yang, 2013).

\section{ESTADO COMERCIAL Y}

\section{TECNOLÓGICO ACTUAL EN INTERFACES HÁPTICAS}

El desarrollo en interfaces hápticas, tanto a nivel cinestésico como táctil, han comenzado a pasar de los laboratorios al usuario final. En este sentido es importante analizar el comportamiento de cada uno de los tipos de interfaces a nivel comercial y las nuevas propuestas que se gestionan a partir de las necesidades técnicas y comerciales.

\section{Estado de difusión comercial de las} interfaces hápticas

A nivel mundial existen diversas empresas que están relacionadas con el desarrollo de tecnologías para las IH, en aspectos tales como la programación (librerías o aplicaciones), componentes electrónicos, mecánicos, sensores, actuadores, y dispositivos hápticos como tal. En el panorama actual (Worldhaptics, 2012), se muestra que hay un buen número de empresas, mayormente en Estados Unidos, que se dedican a la fabricación de IH, además que no hay una participación de países de África, Centro y Suramérica, como se aprecia en la Figura 1.

Figura 1. Distribución de las empresas de interfaces hápticas a nivel mundial

Fuente: elaboración propia

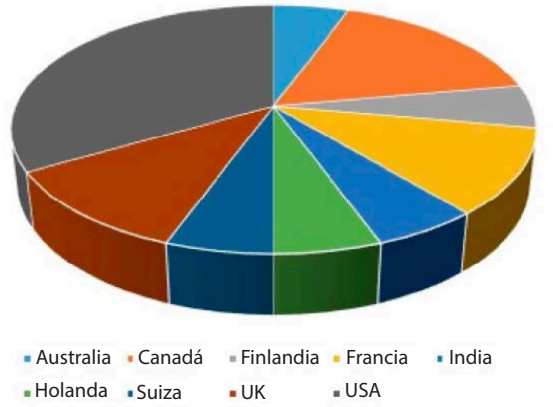

Estas empresas se pueden agrupar también según el tipo de desarrollo más común de tecnolo- gía de simulación del efecto que realiza la IH sobre el usuario, y se clasifican en vibrotáctil, deformación de piel, estiramiento de piel, electroestimulación, térmica y cinestésica, como se aprecia en la Figura 2.

Figura 2. Tecnologías de simulación para las IH Fuente: elaboración propia

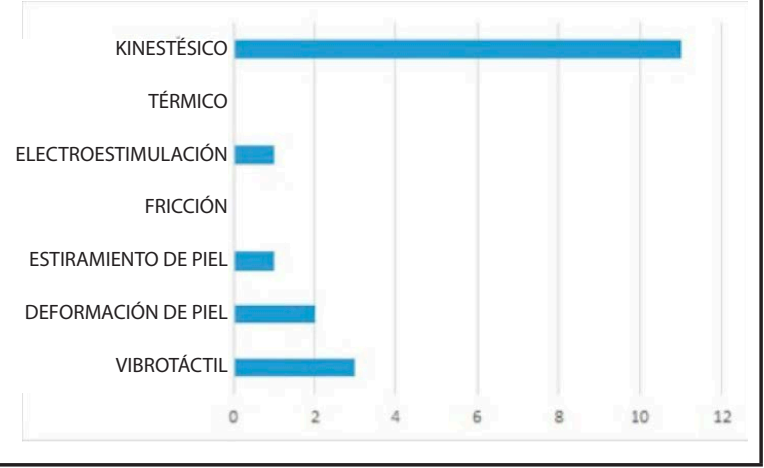

La Tabla 3 resume las IH cinestésicas que se encuentran en el mercado y han logrado mayor difusión en los usuarios.

La Tabla 4 presenta un resumen de los dispositivos táctiles más difundidos en el mercado.

\section{COMBINACIÓN DE TIPOS DE}

\section{INTERFACES}

Las IH tanto táctiles como cinestésicas han logrado un gran desarrollo en los últimos años, pero cada una ha tomado su propio camino. La experiencia del diario trabajo en el área háptica ha demostrado que esta división no es ni efectiva ni intuitiva, ya que la estimulación simultánea de la parte táctil y la cinestésica es esencial (Zeng et al., 2010). Al explorarse un objeto con los dedos directamente, involucra la estimulación tanto de los receptores propioceptivos como los mecanorreceptores cutáneos; los primeros son relacionados con el seguimiento de contornos y los segundos se basan en la presión o distorsión producida en el dedo al contacto con los objetos (Frisoli et al., 2011). Sabiendo que dichos sentidos en el usuario nunca podrán separarse, se entiende con esto que son complementarios o concurrentes y están involucrados cada vez que se esté explorando un objeto.

Revista EIA Rev.ElA.Esc.Ing.Antioq/Universidad EIA 


\begin{tabular}{|c|c|c|c|c|}
\hline Dispositivo & Compañía & $\begin{array}{l}\text { Zona de } \\
\text { acción }\end{array}$ & $\begin{array}{l}\text { Grados de } \\
\text { libertad }\end{array}$ & Principio de funcionamiento y características \\
\hline $\begin{array}{l}\text { PHANTOM } \\
\text { (Sensable 2016) }\end{array}$ & Sensable & $\begin{array}{l}\text { Mano, pivote } \\
\text { en la muñeca }\end{array}$ & 6 & $\begin{array}{l}\text { Morfología serial, primer grado de libertad activo y los } \\
\text { tres últimos pasivos, resolución de posición } 1100 \text { dpi. }\end{array}$ \\
\hline $\begin{array}{l}\text { Omni'M Bundle } \\
\text { (Quanser 2016a) }\end{array}$ & Quanser & Dedos, mano & 6 & $\begin{array}{l}\text { Movimiento de la mano y pivote de muñeca, Fuerza } \\
\text { máxima } 3,3 \mathrm{~N} \text {, espacio de trabajo } 160 \mathrm{~mm} \times 120 \mathrm{~mm} \times \\
70 \mathrm{~mm} \text {. }\end{array}$ \\
\hline $\begin{array}{l}\text { Quanser Haptic 3-DOF } \\
\text { Planar Pantograph } \\
\text { Blocks } \\
\text { (Quanser 2016b) }\end{array}$ & Quanser & Mano & 3 & $\begin{array}{l}\text { Movimiento planar de la mano sujeta a una empuña- } \\
\text { dura, manejado por motores DC. La posición y orien- } \\
\text { tación del efector final del robot como un vector de } 3 \text { : } \\
\text { coordenadas } X \text { e } Y \text { en milímetros, y la orientación, } \theta \text {, en } \\
\text { radianes. }\end{array}$ \\
\hline $\begin{array}{l}\text { NOVINT FALCON } \\
\text { (Novint 2012) }\end{array}$ & Novint & $\begin{array}{l}\text { Dedos, mo- } \\
\text { vimiento de } \\
\text { antebrazo }\end{array}$ & 3 & $\begin{array}{l}\text { Morfología paralela, espacio de trabajo } 4^{\prime \prime} \times 4^{\prime \prime} \text { × } 4 \text { ", fuer- } \\
\text { za } 8.9 \mathrm{~N} \text {, resolución de posición } 400 \text { dpi, calibración au- } \\
\text { tomática. }\end{array}$ \\
\hline $\begin{array}{l}\text { Thrustmaster TX Racing } \\
\text { Wheel Ferrari } 458 \\
\text { (Thrustmaster 2015) }\end{array}$ & Immersion & Manos & 1 & $\begin{array}{l}\text { Volante que proporciona la fuerza para maniobras de } \\
\text { conducción en juego de carreras de autos. }\end{array}$ \\
\hline $\begin{array}{l}\text { Haptic Master } \\
\text { (Mimics 2015) }\end{array}$ & Mimics & Brazos & 3 & $\begin{array}{l}\text { Brazo cinestésico para rehabilitación de movimiento } \\
\text { apoyado en un ambiente virtual. }\end{array}$ \\
\hline $\begin{array}{l}6 \text { Dof DELTA } \\
\text { (Forcedimension 2015a) }\end{array}$ & $\begin{array}{l}\text { Force Di- } \\
\text { mension }\end{array}$ & Dedos, mano & 6 & $\begin{array}{l}\text { Estructura paralela, } 20 \mathrm{~N} \text {, espacio de trabajo semiesféri- } \\
\text { co } \varnothing 400 \times 260 \mathrm{~mm} \text {, rotación } 22^{\circ} \text {, resolución } 0,02 \mathrm{~mm} \text {, } \\
\text { USB } 2.0 \text {, calibración automática. }\end{array}$ \\
\hline $\begin{array}{l}\text { CYBERGRASP } \\
\text { (CybergloveSystems } \\
\text { 2015a) }\end{array}$ & $\begin{array}{l}\text { Cyberglove } \\
\text { Systems }\end{array}$ & Dedos, mano & 5 & $\begin{array}{l}\text { Un grado de libertad por cada dedo } \\
18 \text { a } 22 \text { sensores de fuerza. } \\
\text { Sensores para medir flexión y abducción. }\end{array}$ \\
\hline $\begin{array}{l}\text { HIRO III } \\
\text { (Robothand 2015) }\end{array}$ & Robothand & Dedos & 21 & $\begin{array}{l}6 \text { grados de libertad en el brazo y } 15 \text { en la mano. } \\
\text { Sensación de fuerza y tacto en todas las yemas de los } \\
\text { dedos, apoyo en el suelo. }\end{array}$ \\
\hline $\begin{array}{l}\text { VIRTUOSETM } 6 \text { D Desktop } \\
\text { (Haption 2015) }\end{array}$ & Haption & Dedos, mano & 6 & $\begin{array}{l}\text { Espacio de trabajo } 521 \times 370 \times 400 \mathrm{~mm}, 270^{\circ} \times 120^{\circ} \times \\
250^{\circ} \text {, fuerza máxima traslación } 10 \mathrm{~N} \text {, de rotación } 0,8 \mathrm{~N} \text {, } \\
\text { ethernet/UDP. }\end{array}$ \\
\hline $\begin{array}{l}\text { OMEGA7 } \\
\text { (Forcedimension 2015b) }\end{array}$ & $\begin{array}{l}\text { Force } \mathrm{Di}- \\
\text { mension }\end{array}$ & Mano & 3 & $\begin{array}{l}\text { Estructura paralela, } 8 \mathrm{~N}, \text { USB } 2.0 \text {, rotación } 240 \times 140 \times \\
180^{\circ} \text {. }\end{array}$ \\
\hline $\begin{array}{l}\text { Maglev } 200^{\mathrm{TM}} \\
\text { (Butterfly-haptics 2015) }\end{array}$ & $\begin{array}{l}\text { Butterfly } \\
\text { Haptics }\end{array}$ & Mano & 6 & $\begin{array}{l}\text { Sistema que emplea el principio de levitación de Lo- } \\
\text { rentz para la interacción de fuerzas. }\end{array}$ \\
\hline $\begin{array}{l}\text { SPIDAR } \\
\text { (Sato 2002) } \\
\text { SPIDAR G\&G0 } \\
\text { (Murayama et al., 2004) }\end{array}$ & $\begin{array}{l}\text { Instituto } \\
\text { Tecnológico } \\
\text { de Tokio }\end{array}$ & Mano & 6 & $\begin{array}{l}\text { Basado en motor DC, cables de acero delgado que refle- } \\
\text { jan fuerzas en los efectores finales. Contacto con super- } \\
\text { ficie de objeto. } \\
3 \text { grados de libertad para traslación, } 3 \text { para rotación y } \\
\text { uno para agarre. } \\
\text { - Para dos manos }\end{array}$ \\
\hline $\begin{array}{l}7 \text { DOF Haptic Interface } \\
\text { (MPB 2014) }\end{array}$ & $\begin{array}{l}\text { MPB Tech- } \\
\text { nologies }\end{array}$ & Dedos, mano & 7 & $\begin{array}{l}\text { Para una mano, tijera y platina para dedo índice, espacio } \\
\text { de trabajo traslación: } 17 \times 22 \times 33 \mathrm{~cm}, 2,5 \mathrm{~N} \text {; P-Y-R: } 170^{\circ}- \\
130^{\circ}-340^{\circ} \text {, torque } 370 \mathrm{mN}-310 \mathrm{mN}-150 \mathrm{mN} \text {; tijeras } 40^{\circ} \text { a } \\
450 \mathrm{mN} \text {. }\end{array}$ \\
\hline $\begin{array}{l}\text { DLR light-weight robot III } \\
\text { (DLR 2015) }\end{array}$ & $\begin{array}{l}\text { DLR - Ro- } \\
\text { botics and } \\
\text { Mechatron- } \\
\text { ics Center }\end{array}$ & Mano & 7 & $\begin{array}{l}\text { Máxima carga } 14 \mathrm{Kg} \text {, Ejes R-P-R-P-R-P-P, alcance máximo } \\
936 \mathrm{~mm} \text {, velocidad de juntura } 120^{\circ} / \mathrm{s} \text {, sensor de torque. }\end{array}$ \\
\hline
\end{tabular}




\begin{tabular}{|c|c|c|c|c|c|}
\hline Dispositivo & Compañía & $\begin{array}{l}\text { Zona de } \\
\text { contacto } \\
\text { con el } \\
\text { cuerpo }\end{array}$ & Actuador & Estímulo & Sensación táctil \\
\hline $\begin{array}{l}\text { CYBERTOUCH } \\
\text { (CybergloveSystems } \\
\text { 2015b) }\end{array}$ & $\begin{array}{l}\text { Cyberglove } \\
\text { Systems }\end{array}$ & $\begin{array}{l}5 \text { dedos y } \\
\text { palma de la } \\
\text { mano }\end{array}$ & vibrotáctil & $\begin{array}{l}\text { Vibración 0-125 Hz } \\
1.2 \mathrm{~N}\end{array}$ & Contacto con los objetos. \\
\hline $\begin{array}{l}\text { MI Mannequin Trainer } \\
\text { (MerkelHapticSystems } \\
\text { 2011) }\end{array}$ & $\begin{array}{l}\text { Merkel } \\
\text { Haptics }\end{array}$ & Manos & vibrotáctil & $\begin{array}{l}\text { Simulación de los } \\
\text { signos vitales }\end{array}$ & $\begin{array}{l}\text { Permite el entrenamiento de } \\
\text { las técnicas de Reanimación } \\
\text { Cardio Pulmonar (RCP). }\end{array}$ \\
\hline $\begin{array}{l}\text { HAPIfork } \\
\text { (Hapi 2015) }\end{array}$ & Hapi Labs & Manos & vibrotáctil & $\begin{array}{l}\text { Vibración } \\
\text { moderada }\end{array}$ & $\begin{array}{l}\text { Estímulo en la mano } \\
\text { indicando que se come } \\
\text { rápido. }\end{array}$ \\
\hline $\begin{array}{l}\text { Lumo Lift } \\
\text { (Lumobodytech 2015) }\end{array}$ & $\begin{array}{l}\text { Lumo } \\
\text { BodyTech }\end{array}$ & $\begin{array}{l}\text { Piel del } \\
\text { pecho }\end{array}$ & vibrotáctil & $\begin{array}{l}\text { Vibración } \\
\text { moderada }\end{array}$ & $\begin{array}{l}\text { Estímulo en el pecho que } \\
\text { indica que se está en mala } \\
\text { postura, no erguido. }\end{array}$ \\
\hline $\begin{array}{l}\text { TACTUS Tactile Layer } \\
\text { (TactusTechnology 2015) }\end{array}$ & $\begin{array}{l}\text { Tactus } \\
\text { Technology }\end{array}$ & Dedos & táctil & $\begin{array}{l}\text { Elevación de la } \\
\text { superficie por } \\
\text { medio de un fluido }\end{array}$ & $\begin{array}{l}\text { Presencia de un botón sobre } \\
\text { la pantalla táctil para ser } \\
\text { activado. }\end{array}$ \\
\hline $\begin{array}{l}\text { Reactive Grip }{ }^{\mathrm{TM}} \text { Motion } \\
\text { Controller } \\
\text { (TacticalHaptics 2013) }\end{array}$ & $\begin{array}{l}\text { Tactical } \\
\text { Haptics }\end{array}$ & Mano & táctil & $\begin{array}{l}\text { Estiramiento de } \\
\text { la piel } \\
\text { Movimiento de } \\
\text { placas en el mango } \\
\text { del dispositivo de } \\
\text { forma coordinada }\end{array}$ & $\begin{array}{l}\text { Transmite la información } \\
\text { del movimiento y la fuerza } \\
\text { utilizando retroalimentación } \\
\text { táctil integrada en el mango } \\
\text { del dispositivo. }\end{array}$ \\
\hline $\begin{array}{l}\text { Senseg Tixel } \\
\text { (Senseg 2015) }\end{array}$ & Senseg & Dedos & táctil, & $\begin{array}{l}\text { Fricción en el } \\
\text { dedo por efecto } \\
\text { de fuerzas de } \\
\text { Coulomb }\end{array}$ & $\begin{array}{l}\text { Usa el principio de atracción } \\
\text { de cargas eléctricas, en el } \\
\text { dedo y la pantalla, que entre } \\
\text { sí conforman un capacitor. }\end{array}$ \\
\hline
\end{tabular}

Es por eso que el estímulo táctil y el cinestésico deben ejercerse de manera combinada. Esta situación se está convirtiendo en tendencia en los trabajos realizados en los últimos años, en los que las interfaces táctiles están siendo adaptadas a las interfaces cinestésicas y como resultado se están logrando implementar nuevas técnicas que adaptan las bondades de ambos sistemas para lograr mejores desempeños, evaluados desde diferentes puntos de vista.

Un proyecto de investigación realizado que combina una IH táctil con una IH cinestésica se hizo para la palpación de textiles en forma remota, en el cual se agrupó un actuador táctil de agujas a un dis- positivo de realimentación de fuerza que mostraba por pantalla la interacción del usuario con el textil virtual, con lo que se lograba percibir la sensación de textura y de su inercia (Unige et al., 2008).

El otro caso particular para una IH táctil es la de combinarse con una IH cinestésica cuyo efector final se sitúa en un punto, para convertirla en una IH táctil multipunto (Minamizawa et al., 2010); el mismo principio se ha aplicado en combinación con una o dos interfaces cinestésicas comerciales enfocado a mejorar la estabilidad en un ambiente de teleoperación (Sarakoglou et al., 2012; Pacchierotti et al., 2013). 
Se han dado casos particulares de aplicación de una IH táctil. Uno es el de simular hasta cierto punto la realimentación de fuerza. El método consiste en acoplar un manipulador tipo mango no actuado que soporta la mano y un actuador táctil en los dedos. El mango permite trazar la posición de la mano, mientras que el actuador táctil simula la fuerza de contacto entre la mano y el manipulador por la aplicación de una fuerza normal en la yema de los dedos; la base de esto es que el dispositivo táctil realimenta la fuerza en igual intensidad y forma sobre el área de contacto, como lo haría un mango activo (Pacchierotti et al., 2013; Prattichizzo et al., 2010).

Un enfoque novedoso de la combinación de los tipos de IH es en el que un dispositivo genera estímulo cinestésico sin enlaces mecánicos ni referencia de suelo, pero tienen como inconveniente una menor resolución del estímulo generado. Un primer ejemplo de esto lo es una interfaz cuya forma es de tipo lapicero, la cual tiene la base de referencia en la mano y se sujeta al dedo, sobre el que se genera un estímulo de apretón desde el dedo hacia la base, lo que simula el objeto con el que se interactúa (Kamuro et al., 2011).

Otro ejemplo de la implementación de dicho enfoque es para los dispositivos móviles que se basan en la técnica de "fuerza de pseudo atracción", en la que se genera una sensación de fuerza con un estímulo vibrotáctil por medio de la combinación de patrones de aceleración en dos direcciones para crear la ilusión de una fuerza desbalanceada (Amemiya et al., 2010).

\section{DISCUSIÓN}

Los sistemas cinestésicos permiten la interacción de fuerza con un objeto, tienen la ventaja de mayor facilidad en su construcción pero no logran percibir sensaciones de la superficie de los mismos; de hecho, las interfaces cinestésicas alcanzan la percepción de un objeto en tres dimensiones que se lleva a cabo por la interacción de fuerzas entre la interfaz y el usuario mediante el ambiente virtual en el que se encuentre (Bergamasco y Ruffaldi, 2011).
El gran despliegue que tienen estas interfaces es debido a que se construyen con tecnología convencional (Tabla 1), lo cual permite que se desarrollen y comercialicen con mayor difusión. Lo anterior se evidencia en la amplia gama de dispositivos cinestésicos puestos en el mercado (Tabla 3) y en la presencia de las empresas fabricantes en el primer mundo (Figura 1) (Worldhaptics, 2012). Sus aplicaciones van desde procesos de rehabilitación y teleoperación hasta el área de entretenimiento, validando su aplicabilidad y ventajas comerciales.

Los sistemas táctiles permiten mayor definición de las características de la superficie de los objetos porque trabajan directamente sobre los receptores de la piel, pero el proceso tecnológico de construcción es más complejo y aumenta en razón a las variables a reproducir.

Las interfaces táctiles se dedican a reproducir el contacto con la piel por medio de acciones de presión, de vibración (Chouvardas et al., 2008) y de electro-estimulación (Kajimoto et al., 2004). Con estas se logran estimular los mecano-receptores cutáneos creando sensaciones de textura, contorno y rigidez (Bilgincan et al., 2010; Ferre et al., 2008). Esto se logra por medio de diversas tecnologías para actuadores, que incluyen motores DC, vibro resonadores, matriz de agujas, solenoides y otras más elaboradas como actuadores de láminas piezoeléctricas mono o multicapa, alambres con memoria (SMA), polímeros electro activos (EAP), fluidos electro reorológicos (ERF) y magneto reorológicos (MRF), ultrasonido y electrostática, de los cuales varios de ellos se encuentran en etapa de desarrollo experimental y perfeccionamiento (Tabla 2) (Pasquero, 2006).

Se han logrado interfaces táctiles de alta resolución (Simeonov y Simeonova, 2014) que aumentan el nivel de realismo que el usuario pueda lograr en un ambiente simulado, pero la construcción de estas interfaces es compleja debido a la densa distribución de las terminaciones nerviosas de la piel, lo que exige que el estímulo sea más zonificado al aplicarlo. Una consecuencia a todo esto se evidencia en la menor cantidad de desarrollos de interfaces 
hápticas puestas en el mercado (ver Tabla 4), en contraste con la cantidad de técnicas propuestas para la generación de estímulos táctiles (Tabla 2). Como complemento se presentan algunas dificultades técnicas en cuanto a tamaño y portabilidad, dado que si se desea reproducir dos parámetros táctiles simultáneos, redunda en baja resolución del efecto producido por uno de los estímulos (Fontana et al., 2012). Esta situación se ha ido solucionando de a poco y no se ha alcanzado un punto medio de equilibrio.

Al tener en un mismo dispositivo las bondades de una interfaz cinestésica y una interfaz táctil se obtiene mayor realismo en la interacción con el ambiente virtual-remoto. La combinación de estímulos de las interfaces táctiles y cinestésicas logra mejores resultados, dado que se complementan y aumentan la sensación de realismo, para representar textura espacial y forma contorno, como una superficie plana o curva (Zeng et al., 2010). Al combinar una IH cinestésica y una táctil aumenta la fidelidad en la interacción con el sistema virtual/remoto y mejora la percepción de realismo en el usuario, lo que potencialmente incrementa el nivel de inmersión virtual/remota (García-Hernández et al., 2014) que se evidencia también en la disminución del error de orientación en tareas de palpación (Unige et al., 2008). La materialización de este hecho se ha realizado con facilidad con la combinación entre una interfaz cinestésica comercial y una interfaz táctil desarrollada para el dedo, con lo que han logrado resultados satisfactorios (Minamizawa et al., 2010; Sarakoglou et al., 2012; Pacchierotti et al., 2013). Existe otro tipo de combinación en donde por medios táctiles se logran efectos cinestésicos simulados y sin enlaces o referencia de suelo, por medio de la combinación de impulsos de fuerza-torque que aplicados en la piel de la mano, dan la sensación de manipulación de objetos (Prattichizzo et al., 2010) (Kamuro et al., 2011; Amemiya et al., 2010).

Estos argumentos finales evidencian la tendencia percibida, con base en los desarrollos en los últimos años, que por combinar los efectos producidos por ambos tipos de interfaces hápticas, la inte- racción del usuario con objetos en ambientes virtuales/remotos produce un mayor grado de inmersión en la manipulación de los mismos, con lo que se consigue favorecer aspectos útiles a las tareas particulares, tales como mejorar destrezas, reducir curvas de aprendizaje, en tanto el sistema se esté utilizando como medio para aprendizaje de una tarea de habilidad manual. Este hecho se puede hacer extensivo si se aplica con la perspectiva de rehabilitación de personas con alguna lesión motriz en extremidades superiores, tal como se enfoca el concepto de juego serio (Bouri, 2013).

\section{CONCLUSIONES}

Existe una tendencia en combinar sistemas cinestésicos con sistemas táctiles para lograr mayor realismo e inmersión en la percepción de las sensaciones en la interacción con objetos en un ambiente virtual o remoto. Se percibe que las tecnologías utilizadas para fabricación de IH cinestésicas son más limitadas pero a la vez más conocidas, convencionales y por tanto las más implementadas. Las interfaces táctiles presentan mayor variedad en la forma y tecnología de construcción, pero a su vez los desarrollos están en la mayoría de los casos en fases experimentales y no comerciales, con aspectos por mejorar y refinar, debido a que el estímulo va dirigido receptores nerviosos densamente ubicados en la piel y de percepción específica.

Se hallan evidencias de que es posible realizar un acople de IH combinadas haciendo adaptaciones de desarrollos táctiles a interfaces cinestésicas comerciales, basadas en que se han realizado combinaciones de IH cinestésicas y táctiles que han permitido resultados satisfactorios en la representación de textura e inercia de movimiento, al igual que contacto y manipulación de objetos. Esta combinación de los tipos de IH tiene potencial para ser utilizada en múltiples aplicaciones donde sea útil la identificación simultánea de textura y contorno.

En el aspecto del aumento del grado de inmersión, se continúa trabajando tanto en las interfaces táctiles como cinestésicas, ya que requieren 
adecuaciones, adaptaciones y combinación de nuevas tecnologías para la mejora de la resolución, disminución de tamaño y portabilidad de los sistemas.

\section{REFERENCIAS}

Amemiya, T.; Ando, H.; Maeda, T. (2010). Kinesthetic Illusion of Being Pulled Sensation Enables Haptic Navigation for Broad Social Applications. In M. Hosseini Zadeh, ed. Advances in Haptics. InTech, pp. 129-134. [Online] Disponible en: http://goo.gl/bI6dOf.

Benali-Khoudja, M.; Hafez, M.; Alexandre, J.-M. (2004). Tactile interfaces: a state-of-the-art survey. ISR 2004, 35th International Symposium on Robotics, pp. 23-26.

Bergamasco, M.; Ruffaldi, E. (2011). Haptic Interfaces for Embodiment in Virtual Environments. Proceedings of RO-MAN 2011. [Online] Disponible en: http:// goo.gl/mL08Jw.

Berkelman, P.; Dzadovsky, M. (2010). Using Magnetic Levitation for Haptic Interaction. Advances in Haptics, pp.31-46. [Online] Disponible en: http://goo.gl/ GjHFaa.

Bianchi, M.; Gwilliam, J.C.; Degirmenci, A.; Okamura, A.M. (2011). Characterization of an Air-Jet Haptic Lump Display. Proceedings IEEE Engineering in Medicine and Biology Society, (Query date: 2015-07-24). [Online] Disponible en: http://goo.gl/nMQjWG.

Bianchi, M.; Serio, A.; (2015). Design and characterization of a fabric-based softness display. IEEE Transactions on Haptics, 8(2), pp. 152-163.

Bianchi, M.; Valenza, G.; Serio, A., Lanatà, A.; Greco, A.; Nardelli, M.; Scilingo, E.P.; Bicchi, A. (2014). Design and preliminary affective characterization of a novel fabric-based tactile display. IEEE Haptics Symposium, HAPTICS, pp. 591-596.

Bilgincan, T.; Gezgin, E.; Mehmet, İ.; Dede, C. (2010). Integration of the Hybrid-Structure Haptic Interface : HIPHAD v1 . 0. Proceedings of the International Symposium of Mechanism and Machine Theory, p. 18.

Bolzmacher, C.; Hafez, M.; Khoudja, M.B.; Bernardoni, P.; Dubowsky, S. (2004). Polymer-based actuators for virtual reality devices. Smart Structures and Materials: Electroactive Polymer Actuators and Devices (EAPAD), 5385, pp. 281-289. [Online] Disponible en: http://goo.gl/jv7J7P.
Bouri, M.; Baur, C.; Clavel, R.; Zedka, M.; Newman, C.J. (2013). "Handreha": A new Hand and Wrist Haptic Device for Hemiplegic Children. ACHI 2013: The Sixth International Conference on Advances in Computer-Human Interactions "Handreha":, pp. 286-292.

Butterfly-haptics (2015). Maglev 200 ${ }^{\mathrm{TM}}$ System. [Online] Disponible en: http://butterflyhaptics.com/ products/system/ [Consultado 1 de noviembre de 2015].

Carter, J.; Fourney, D. (2005). Research Based Tactile and Haptic Interaction Guidelines. Guidelines On Tactile and Haptic Interactions Conference (GOTHI-05), pp. 84-92. [Online] Disponible en: http://userlab. usask.ca/GOTHI/research.pdf.

Chen, L.J.; Holbein, M.; Zelek, J.S. (2006). Intro to haptic communications for high school students. Proceedings - IEEE International Conference on Robotics and Automation, 2006(May), pp. 733-738.

Chinello, F.; Malvezzi, M.; Pacchierotti, C.; Prattichizzo, D. (2015). Design and development of a 3RRS wearable fingertip cutaneous device. IEEE/ASME International Conference on Advanced Intelligent Mechatronics, AIM, 2015-Augus, pp. 293-298.

Chouvardas, V.G.; Miliou, A.N.; Hatalis, M.K. (2008). Tactile displays: Overview and recent advances. Displays, 29(3), pp. 185-194.

Coles, T.R. (2011). Investigating Augmented Reality VisioHaptic Techniques for Medical Training (PhD). Prifysgol Bangor University. [Online] Disponible en: http://goo.gl/g9hYjY. [Consultado 10 de noviembre de 2015].

Coles, T.R.; Meglan, D.; John, N.W. (2011). The role of haptics in medical training simulators: A survey of the state of the art. IEEE Transactions on Haptics, 4(1), pp. 51-66.

Cortés, H.H.; México, C.; García, M.A. (2010). Aplicaciones para Entornos Educativos. SISTEMAS, CIBERNÉTICA E INFORMÁTICA, 7, pp. 46-49. [Online] Disponible en: https://goo.gl/koKxul.

CybergloveSystems (2015a). Cybergrasp. [Online] Disponible en: http://www.cyberglovesystems.com/ cybergrasp/ [Consultado 1 de noviembre de 2015].

CybergloveSystems (2015b). Cybertouch. [Online] Disponible en: http://www.cyberglovesystems.com/ cybertouch/ [Consultado 1 de noviembre de 2015]. 
DLR (2015). DLR light-weight robot III. [Online] Disponible en: http://www.dlr.de/rmc/rm/desktopdefault. aspx/tabid-3803/6175_read-8963/ [Consultado 1 de noviembre de 2015].

Do, P.; Homa, D.; Ferguson, R.; Crawford, T. (2012). Haptic Concepts. In A. El Saddik, ed. Haptics Rendering and Applications. Arizona: InTech, pp. 3-24. [Online] Disponible en: http://goo.gl/TfVMPY.

ElSaddik, A.; Orozco, M.; Eid, M.; Cha, J. (2011). Haptics: General Principles. In Haptics Technologies. Springer, pp.1-21. [Online] Disponible en: http://goo.gl/ Ytv9He.

Evreinova, T. V.; Evreinov, G.; Raisamo, R. (2014). From Kinesthetic Sense to New Interaction Concepts: Feasibility and Constraints. International Journal of Advanced Computer Technology, 3(4), pp. 1-33. [Online] Disponible en: http://goo.gl/QhjZG2.

Ferre, M.; Oyarzábal, M.; Campos, A.; Monroy, M. (2008). Multifinger Haptic Interfaces for Collaborative Enviroments. In I. Pavlidis, ed. Human-Computer Interaction. InTech, p. 522. [Online] Disponible en: http:// goo.gl/WFXVCT.

Fogtmann, M.H.; Fritsch, J.; Kortbek, K.J. (2008). Kinesthetic Interaction - Revealing the Bodily Potential in Interaction Design. Proceedings of the 20th Australasian Conference on ComputerHuman Interaction Designing for Habitus and Habitat, pp. 89-96. [Online] Disponible en: http://goo.gl/xaTGh2.

Fontana, M.; Ruffaldi, E.; Salasedo, F.; Bergamasco, M. (2012). On the Integration of Tactile and Force Feedback. In A. El Saddik \& ISBN, eds. Haptics Rendering and Applications. InTech, pp. 47-75. [Online] Disponible en: http://goo.gl/Txh3tz.

Forcedimension (2015a). delta.6. [Online] Disponible en: http://www.forcedimension.com/products/ delta-6/overview [Consultado 1 de noviembre de 2015].

Forcedimension (2015b). omega.7. [Online] Disponible en: http://www.forcedimension.com/products/ omega-7/overview [Consultado 1 de noviembre de 2015].

Frisoli, A.; Solazzi, M.; Reiner, M.; Bergamasco, M. (2011). The contribution of cutaneous and kinesthetic sensory modalities in haptic perception of orientation. Brain Research Bulletin, 85(5), pp. 260-266. [Online] Disponible en: http://dx.doi.org/10.1016/j.brainresbull.2010.11.011.
García-Hernández, N.; Bertolotto, F.; Cannella, F.; Tsagarakis, N.G.; Caldwell, D.G. (2014). How tactor size and density of normal indentation tactile displays affects grating discrimination tasks. IEEE Transactions on Haptics, 7(3), pp. 356-366.

Genoy Muñoz, J.W.; Rodríguez Ortiz, L.F.; Salinas, S.A. (2011). Interfaz háptica de cuatro grados de libertad para aplicaciones quirúrgicas. Revista Ingeniería Biomédica, 5(9), pp. 35-42.

Geomagic (2015). Geomagic $₫$ Haptic Devices. [Online] Disponible en: http://www.geomagic.com/en/ products-landing-pages/haptic [Consultado 1 de noviembre de 2015].

Golledge, R.G.; Rice, M.T.; Jacobson, R.D. (2006). Multimodal interfaces for representing and accessing geospatial information. Frontiers of Geographic Information Technology, p. 38.

González, L. (2011). Visión global sobre tecnología háptica. Revista ACTA, 61, pp. 115-122. [Online] Disponible en: http://goo.gl/AkxWtd.

Hapi (2015). HAPIfork. [Online] Disponible en: https:// www.hapi.com/product/hapifork [Consultado 1 de noviembre de 2015].

Haption (2015). VIRTUOSE TM 6D Desktop. [Online] Disponible en: http://www.haption.com/site/index. php/en/products-menu-en [Consultado 1 de noviembre de 2015].

Hernantes, J.; Díaz, I.; Borro, D.; Gil, J. (2012). Effective Haptic Rendering Method for Complex Interactions. In A. El Saddik, ed. Haptics Rendering and Applications. InTech, p. 246. [Online] Disponible en: http:// goo.gl/LNCJ8W.

Hoshi, T.; Takahashi, M.; Iwamoto, T.; Shinoda, H. (2010). Noncontact tactile display based on radiation pressure of airborne ultrasound. IEEE Transactions on Haptics, 3(3), pp. 155-165.

Iwamoto, T.; Shinoda, H. (2005). Ultrasound Tactile Display for Stress Field Reproduction -Examination of Non-Vibratory Tactile Apparent Movement-. Proceedings of World Haptics 2005, (Ultrasound Tactile Display), pp. 220-228.

Kajimoto, H.; Kawakami, N.; Maeda, T.; Tachi, S. (2004). Electro-tactile display with tactile primary color approach. Proceedings of International Conference on Intelligent Robots and Systems, 10.

Kamuro, S.; Minamizawa, K.; Tachi, S. (2011). An ungrounded pen-shaped kinesthetic display: Device cons-

Revista EIA Rev.EIA.Esc.Ing.Antioq / Universidad EIA 
truction and applications. 2011 IEEE World Haptics Conference, WHC 2011, pp.557-562.

Kontarinis, D.A.; Son, J.S.; Peine, W.; Howe, R.D. (1995). A tactile shape and sensing display system for teleoperated manipulation. IEEE International Conference on Robotics and Automation, pp. 641-646. [Online] Disponible en: https://goo.gl/fdVxUA.

Kyung, K.; Kwon, D. (2008). Tactile Displays with Parallel Mechanism. In J.-H. Ryu, ed. Parallel Manipulators, New Developments. InTech, p. 498. [Online] Disponible en: http://goo.gl/3aRSzD.

Lee, J.S.; Lucyszyn, S. (2005). A micromachined refreshable Braille cell. Journal of Microelectromechanical Systems, 14(4), pp. 673-682.

Lévesque, V.; Petit, G.; Dufresne, A.; Hayward, V. (2012). Adaptive level of detail in dynamic, refreshable tactile graphics. Haptics Symposium 2012, HAPTICS 2012 - Proceedings, pp. 1-5.

Lim, T.; Germánico, G.B.; Medellín Castillo, H.I. (2014). Are you haptic a bad day. Proceedings of the ASME 2014 International Design Engineering Technical Conferences \& Computers and Information in Engineering Conference IDETC/CIE 2014, pp. 1-9.

López, J.; Galiana, I.; Torres, J.L.; Ferre, M.; Giménez, A. (2011). Análisis y diseño mecánico de una interfaz háptica para tareas de manipulación. In CIBIM 10. Oporto, Portugal, pp. 1633-1640.

Lumobodytech (2015). Lumo Lift. [Online] Disponible en: http://www.lumobodytech.com/product-category/lumo-lift/ [Consultado 1 de noviembre de 2015].

Matysek, M.; Lotz, P.; Winterstein, T.; Schlaak, H.F. (2009). Dielectric Elastomer Actuators for Tactile Displays. Third Joint Eurohaptics Conference and Symposium on Haptic Interfaces for Virtual Environment and Teleoperator Systems Salt, pp. 290-295.

MerkelHapticSystems (2011). MI Mannequin Trainer. [Online] Disponible en: http://www.merkelhaptics. com [Consultado 1 de noviembre 2015].

Mimics (2015). Haptic Master. [Online] Disponible en: http://www.mimics.ethz.ch/index.php [Consultado 1 de noviembre de 2015].

Minamizawa, K.; Prattichizzo, D.; Tachi, S. (2010). Simplified design of haptic display by extending one-point kinesthetic feedback to multipoint tactile feedback. 2010 IEEE Haptics Symposium, HAPTICS 2010, pp. $257-260$.
MPB (2014). 7 DOF Haptic Interface. [Online] Disponible en: http://www.mpb-technologies.ca/mpbt/mpbt web_2009/_en/7dof/specs.html [Consultado 1 de noviembre de 2015].

Murayama, J.; Bougrila, L.; Luo, Y. (2004). SPIDAR G\&G: A two-handed haptic interface for bimanual VR interaction. proceedings of Eurohaptics, pp. 138-146. [Online] Disponible en: http://goo.gl/s80J0g.

Nájera, E.; Díaz, A. (2005). Interacción pseudoháptica en el simulador de entrenamiento APSiDeTH. Simposio Interacción 2005, pp. 97-100. [Online] Disponible en: aipo.es/articulos/5/1374.pdf.

Nakamura, M.; Engineering, B.S.M.; Jones, L.A.; Supervisor, T.; Sonin, A.A. (2003). A Torso Haptic Display based on Shape Memory Alloy Actuators. Thesis. Master of Science in Mechanical Engineering. Massachusetts Institute of Technology.

Naud, M.; Chamaret, D.; Hamon, L.; Ullah, S.; Richard, E.; Richard, P. (2009). Human-Scale Haptic Interaction Using the SPIDAR. VRCO9 - Joint Virtual Reality Conference of EGVE - ICAT -EuroVR (JVRC 2009), 1, pp. 123-128. [Online] Disponible en: https://goo.gl/ I0VpyZ.

Novint (2012). Novint Falcon. [Online] Disponible en: http://www.novint.com/index.php/novintfalcon [Consultado 1 de noviembre de 2015].

O'Malley, M.; Gupta, A. (2008). Haptic Interfaces. In P. Kortum, ed. HCI Beyond the GUI: Design for Haptic, Design for Haptic, Speech, Olfatory and other Nontraditional Interfaces. MK, pp. 25-73.

Pacchierotti, C.; Tirmizi, A.; Bianchini, G.; Prattichizzo, D. (2013). Improving transparency in passive teleoperation by combining cutaneous and kinesthetic force feedback. IEEE International Conference on Intelligent Robots and Systems, p.6.

Pamungkas, D.; Ward, K. (2015). Tactile sensing system using electro-tactile feedback. ICARA 2015 - Proceedings of the 2015 6th International Conference on Automation, Robotics and Applications, p.8.

Pasquero, J. (2006). Survey on communication through touch. Center for Intelligent Machines-McGill University, Tech. Rep. TR-CIM, pp. 1-27. [Online] Disponible en: http://goo.gl/OciBuq.

Pasquero, J.; Hayward, V. (2003). STReSS: A Practical Tactile Display System with One Millimeter Spatial Resolution and $700 \mathrm{~Hz}$ Refresh Rate. Proc Eurohaptics, 
pp. 94-110. [Online] Disponible en: http://goo.gl/ hYPgz9.

Pei, Q.; Yu, Z.; Niu; Xiaofan Brochu, P. (2009). Electroactive polymers for rigid-to-rigid actuation and Braille e-books. Proceedings of SPIE - The International Society for Optical Engineering, pp. 1-6. [Online] Disponible en: http://goo.gl/YaBluM.

Perez, A.G.; Lobo, D.; Chinello, F.; Cirio, G.; Malvezzi, M. (2015). Soft Finger Tactile Rendering for Wearable Haptics. IEEE World Haptics Conference, pp. 327332.

Poupyrev, I.; Maruyama, S.; Rekimoto, J. (2002). Ambient touch: designing tactile interfaces for handheld devices. Proceedings of the 15th annual ACM symposium on User interface software and technology, pp. 51-60. [Online] Disponible en: http://goo.gl/ d07UCD.

Prattichizzo, D.; Pacchierotti, C.; Cenci, S.; Minamizawa, K.; Rosati, G. (2010). Using a fingertip tactile device to substitute kinesthetic feedback in haptic interaction. Lecture Notes in Computer Science (including subseries Lecture Notes in Artificial Intelligence and Lecture Notes in Bioinformatics), pp. 125-130.

Pyo, D.; Yang, T.-H.; Ryu, S.; Kwon, D.-S. (2015). Novel linear impact-resonant actuator for mobile applications. Sensors and Actuators A: Physical, 233, pp. 460-471.

Quanser (2016a). Omni Bundle. [Online] Disponible en: http://www.quanser.com/products/omni_bundle [Consultado 15 de enero de 2016].

Quanser (2016b). Planar Pantograph Blocks. [Online] Disponible en: http://www.quanser.com/ Products/2dof_serial_flexible_joint [Consultado 15 de enero de 2016].

Rantala, J.; Raisamo; Lylykangas, J.; Ahmaniemi; Makela; Salminen; Surakka (2011). The role of gesture types and spatial feedback in haptic communication. Tansactions on Haptics, 4(4), pp. 295-306. [Online] Disponible en: http://goo.gl/e8p07A.

Richter, H.; Blaha, B.; Wiethoff, A. (2011). Tactile feedback without a big fuss: simple actuators for high-resolution phantom sensations. Proceedings of the 13th International Conference on Ubiquitous Computing - UbiComp '11, pp. 85-88. [Online] Disponible en: http://goo.gl/tzaYpm.

Richter, H.; Manke, F.; Seror, M. (2013). LiquiTouch: Liquid As a Medium for Versatile Tactile Feedback on Touch Surfaces. Proceedings of the 7th International
Conference on Tangible, Embedded and Embodied Interaction, pp. 315-318. [Online] Disponible en: http://goo.gl/0rChkZ.

Robothand (2015). Haptic robot HIRO III. [Online] Disponible en: http://robothand.eu/en/products/robotic_hands/haptic_robot_hiro_iii/ [Consultado 1 de noviembre de 2015].

Runyan, N.; Blazie, D. (2010). EAP actuators aid the quest for the "Holy Braille" of tactile displays. Spie, p.12. [Online] Disponible en: http://goo.gl/Q3JvkM.

Russomanno, A.; Gillespie, R.B.; O’Modhrain, S.; Burns, M. (2015). The design of pressure-controlled valves for a refreshable tactile display. IEEE World Haptics Conference, WHC 2015, pp. 177-182.

Sabater, J.M. (2003). Desarrollo de una interfaz paralela y en experimentación en control de sistemas hápticos y teleoperados (Ingeniero de Sistemas). Escuela Politécnica Superior de Elche.

Sarakoglou, I.; García-Hernández, N.; Tsagarakis, N.G.; Caldwell, D.G. (2012). A high performance tactile feedback display and its integration in teleoperation. IEEE Transactions on Haptics, 5(3), pp. 252-263.

Sato, K.; Tachi, S. (2010). Design of electrotactile stimulation to represent distribution of force vectors. 2010 IEEE Haptics Symposium, HAPTICS 2010, pp. 121-128.

Sato, M. (2002). Development of string-based force display: SPIDAR. In Proc. The Eighth International Conference on Virtual Systems and Multi Media VSMM, pp.1034-1039. [Online] Disponible en: http://goo. $\mathrm{gl} / \mathrm{XAJ} 5 \mathrm{bh}$.

Sensable (2016). PHANTOM OMNI® Haptic Device. [Online] Disponible en: http://www.dentsable.com/ haptic-phantom-omni.htm [Consultado 15 de enero de 2016].

Senseg (2015). Senseg Tixel. [Online] Disponible en: http://www.senseg.com [Consultado 1 de noviembre de 2015].

Simeonov, S.; Simeonova, N. (2014). Graphical Interface for Visually Impaired People Based on Bi-stable Solenoids. arXiv preprint arXiv:1401.5289. [Online] Disponible en: http://goo.gl/Jlu3GT.

Sledd, A.; O’Malley, M.K. (2006). Performance Enhancement of a Haptic Arm Exoskeleton. 2006 14th Symposium on Haptic Interfaces for Virtual Environment and Teleoperator Systems, pp. 375-381. 
Song, A.; Liu, J.; Wu, J. (2003). Softness Haptic Display Device for Human- Computer Interaction. In I. Pavlidis, ed. Human-Computer Interaction. InTech, pp. 257279. [Online] Disponible en: http://goo.gl/jDtlA5.

TacticalHaptics (2013). Reactive Grip ${ }^{\mathrm{TM}}$ Motion Controller. [Online] Disponible en: http://tacticalhaptics.com/ products / [Consultado 1 de noviembre de 2015].

TactusTechnology (2015). TACTUS Tactile Layer. [Online] Disponible en: http://tactustechnology.com/technology/ [Consultado 1 de noviembre de 2015].

Thrustmaster (2015). Thrustmaster TX Racing Wheel Ferrari 458. [Online] Disponible en: http://goo.gl/ HR3Uoj [Consultado 1 de noviembre de 2015].

Torres, D. (2012). Diseño de ortoprótesis para mano. Mexico DF: Universidad Nacional Autónoma de México. [Online] Disponible en: http://goo.gl/3T57Xe.

Ueberle, M. (2006). Design, Control, and Evaluation of a Family of Kinesthetic Haptic Interfaces (Doctor - Engineer). Technical University of Munich.

Unige; Unexe; Percro; Uhan; Swl (2008). Whole Haptic Interface Hardware. Report., [Online] Disponible en: http://goo.gl/Kn4VdC.

Vélez, A. (2011). Diseño mecánico de un interfaz háptico para realidad virtual. (Ingeniero Mecánico). Universidad Carlos III de Madrid.

Wagner, C.; Lederman, S.J.; Howe, R.D. (2004). Design and performance of a tactile shape display. Haptics-e, p.6.

Wagner, S.R. (2014). Haptic and Exoskeleton Devices for Neurorehabilitation of Upper Limb Paralysis : a State of Art and a Night Landing Task. Northern Michigan University. [Online] Disponible en: http://goo. gl/75PUOU.

Wang, F. (2014). Haptic Energy Consumption. Application. Report., Dallas, Texas. [Online] Disponible en: http://goo.gl/78bICu.

Worldhaptics (2012). Haptics Technical Commite, Companies. [Online] Disponible en: http://www.worldhaptics.org/companies [Consultado 15 de noviembre de 2015].

Xu, C.; Israr, A.; Poupyrev, I.; Bau, O.; Harrison, C. (2011). Tactile display for the visually impaired using TeslaTouch. Proc. CHI EA '11, pp. 317-322. [Online] Disponible en: http://goo.gl/cDLu5c.

Yang, Y. (2013). Design and Control of an Integrated Haptic Interface for Touch Screen Applications (Doctorat). Université Lille 1 [Consultado 9 de noviem- bre de 2015]. [Online] Disponible en: http://goo. gl/9J8s4R.

Yazdian, S.; Doxon, A.J.; Johnson, D.E.; Tan, H.Z.; Provancher, W.R. (2013). 2-DOF contact location display for manipulating virtual objects. In 2013 World Haptics Conference, WHC 2013. Daejeon, Korea: IEEE, pp. 443-448.

Youngblut, C.; Johnson, R.E.; Nash, S.H.; Wienclaw, R.A.; Will, C.. (1996). Kinesthetic interfaces. In Review of virtual environment interface technology. Alexandria, Virginia: Institute for Defense Analyses, pp. 117-154. [Online] Disponible en: http://goo.gl/ vL5B4B.

Zeng, T.; Giraud, F.; Lemaire-Semail, B.; Amberg, M. (2010). Analysis of a New Haptic Display Coupling Tactile and Kinesthetic Feedback to Render Texture and Shape. In A. M. L. Kappers, J. B. . van Erp, W. M. Bergmann Tiest, \& F. C. T. van der Helm, eds. International Conference, EuroHaptics. Springer, pp. 87-93.

\section{PARA CITAR ESTE ARTÍCULO / TO REFERENCE THIS ARTICLE / PARA CITAR ESTE ARTIGO /}

Pérez-Ariza, V.; Santís-Chaves, M. (2016). Interfaces Hápticas: sistemas cinestésicos vs. sistemas táctiles. Revista EIA, 13(26), julio-diciembre, pp. 13-29. [Online]. Disponible en: https://doi.org/10.24050/reia.v13i26.1065 